\title{
GMR
}

\section{Roles of microRNA-221/222 in type 2 diabetic patients with post-menopausal breast cancer}

\author{
M.Y. Li ${ }^{1}$, S.R. Pan² and A.Y. Qiu ${ }^{1}$ \\ ${ }^{1}$ Department of General Surgery, People's Hospital, Binzhou, \\ Shandong, China \\ ${ }^{2}$ Department of Medical Gastroenterology, People's Hospital, \\ Binzhou, Shandong, China \\ Corresponding author: M.Y. Li \\ E-mail: mengyuli_binzhou@163.com
}

Genet. Mol. Res. 15 (2): gmr.15027259

Received July 21, 2015

Accepted February 29, 2016

Published July 15, 2016

DOI http://dx.doi.org/10.4238/gmr.15027259

\begin{abstract}
The aim of the research was to examine the expression level of microRNA221/222 (miR-221/222) in the serum of patients with type 2 diabetes mellitus (T2DM) who are also diagnosed with post-menopausal breast cancer. We aimed to evaluate the differences in microRNA expression in patients with T2DM alone, patients with postmenopausal breast cancer alone, and patients with both T2DM and postmenopausal breast cancer. We selected 20 cases from a healthy control group, 30 cases from the group of patients with T2DM and obesity, 30 cases from the group of the patients with post-menopausal breast cancer, and 30 cases from the group of patients with both T2DM and post-menopausal breast cancer. The expression of miR-221/222 in the serum of the patients with post-menopausal breast cancer was higher than that of T2DM patients $(\mathrm{P}<0.05)$, but lower than that of the T2DM patients who were also positive for post-menopausal breast cancer ( $\mathrm{P}$ $<0.05)$; the expression of miR-221/222 in the serum of the T2DM patients was higher than that of the healthy controls $(\mathrm{P}<0.05)$. BMI, HOMA-IR, HbA1c, and TG were positively correlated with the relative expression of miR-221/222 in the serum $(\mathrm{P}<0.01)$. In conclusion, miR-
\end{abstract}


$221 / 222$ participates in insulin resistance; the combination of miR$221 / 222$ and estrogen contributes to incidence of T2DM with postmenopausal breast cancer complications. MiR-221/222 may participate in the occurrence and progression of T2DM with post-menopausal breast cancer via down-regulation of CAVl.

Key words: miR-221/222; Diabetes mellitus; Breast cancer

\section{INTRODUCTION}

Breast cancer is an estrogen-dependent cancer. Estrogen exerts its regulation on cell proliferation by binding to the estrogen receptors on target cells (Zhu et al., 2014). The major estrogenic hormones are estradiol and estrone. Following menopause, estrogens are primarily secreted from adipose tissues, and do not exhibit any diurnal or nocturnal cycles. In addition, the metabolic clearance rate of estrogen decreases by $30 \%$ after menopause. It is known that post-menopausal estradiol is mainly converted from estrone. Estrone is converted from androstenedione in the peripheral tissue. Following menopause, the rate of this conversion increases by 1 fold, which primarily takes place in the fat and muscle tissues. Studies have additionally shown that the conversion rate in obese people is higher than that in lean people. Estrogen level is positively correlated with body weight (Du et al., 2014; Reinbothe et al., 2014). After menopause, estrogen level gradually declines while body fat content increases. Studies have shown that the rate of post-menopausal breast cancer is between 39 and $92 \%$, which is higher than that of pre-menopausal breast cancer (Zhang et al., 2013). Estrogen serves as ligand and substrate to stimulate cell proliferation and gene expression via interactions with the estrogen receptor. Furthermore, it induces DNA damage via formation of oxidative products, thus participating in the pathogenesis and progression of breast cancer (García et al., 2010).

Type 2 diabetes mellitus (T2DM) mainly occurs in middle aged and elderly people (after menopause). Insulin resistance is one of the major causes of type 2 diabetes (Tawfik et al., 2015). Present research suggested that T2DM is not correlated with pre-menopausal breast cancer, but is moderately correlated with the post-menopausal breast cancer (Gallagher et al., 2012). In addition, fasting insulin level is also positively correlated with post-menopausal breast cancer. However, the relationship between T2DM and post-menopausal breast cancer is highly debated (Gallagher et al., 2012). Insulin resistance is not only present in T2DM, but also in the patients with malignant tumors, suggesting that the insulin resistance may be a common factor in the pathogenesis of both diseases. It is known that insulin resistance is the pathological basis for T2DM; however, the molecular mechanisms leading to insulin resistance have not been fully explained. It was suggested that abnormal expression of inflammatory factors, oxidative stress, adipose cytokines, and miRNAs can lead to insulin resistance by altering the signal transduction pathway of insulin as well as the function of glucose transcription factor-4 (Roh et al., 2014; Wen et al., 2014). Interestingly, these factors are also present in patients with malignant tumors, which in turn contribute to insulin resistance in patients with malignant tumors. They have been shown to induce damage to intracellular DNA, thus leading to DNA methylation, abnormal gene expression, and participation occurrence of malignant tumors (Roh et al., 2014; Wen et al., 2014). MiR-221 and miR-222 are members of the microRNA family. They differ only by one nucleotide, and are located on different chromosomes in humans. They are expressed in many human organs such as the brain, and participate in the development of metabolic pathway, 
diabetes, and tumors (Dentelli et al., 2014). Abnormal expression of these miRNAs is observed in cases of obesity, cancer, and anoxia. Studies have shown that expression of miR-221/222 is up-regulated in rats and humans with fatty liver disease. They are also positively correlated with the insulin resistance index (Dentelli et al., 2014). Additionally, they are highly expressed in breast cancer tissues, and elevated serum level of miR-221 and miR-222 is correlated with tumor metastasis. Higher expression of miR-221/222 is suggestive of poor breast cancer prognosis, and is used as one of the indexes for prediction of disease progression (Hwang et al., 2013). The survival rate in breast cancer patients with over-expressed miR-221/222 is lower compared with patients with low miR-221/222 lowly expression.

Another factor that plays a role in insulin resistance is caveolin-1. Studies have shown that down-regulation of caveolin-1(CAV1) causes insulin resistance and an increase in glucose export (Rippe et al., 2012). Research has demonstrated that miR-221/222 is highly expressed in the liver of obese mice, and that silencing of miR-221/222 can up-regulate CAV1 expression, improve the insulin sensitivity, and decrease blood glucose (Rippe et al., 2012). CAV1 is one of the main scaffolding proteins of the cytoplasmic membrane, which can stabilize insulin receptors and enhance the function of glucose transcription factor-4. Its abnormal expression can lead to a decline in insulin sensitivity and an increase in blood glucose (Chen et al., 2011). Down-regulation of CAV1 can weaken physiological functions of insulin, reduce insulin sensitivity, lead to reduced sugar tolerance, and even diabetes. Furthermore, mutation or methylation of the CAV1 gene in breast cancer tissues cause deactivation or down-regulation of protein expression, activate estrogen receptor alpha, and trigger the occurrence and progression of breast cancer (Joglekar et al., 2015).

Our study aims to examine the expression levels of miR-221/222 in serum of the patients with T2DM and post-menopausal breast cancer. We wanted to examine expression levels of miR-221/222 in the serum of T2DM patients, and determine their roles in insulin resistance. We also wanted to investigate the difference between patients with T2DM and patients with T2DM and post-menopausal breast cancer, and determine whether CAV-I is a target gene of miR-221/222.

\section{MATERIAL AND METHODS}

\section{Study subjects}

We selected 30 (DM group) patients that were newly diagnosed with post-menopausal breast cancer at Binzhou People's Hospital, and 30 (BC group) patients newly diagnosed with post-menopausal breast cancer, as well as 30 (DM and BC group, hereinafter referred to as DB group) patients newly diagnosed with both T2DM and post-menopausal breast cancer. In addition, 20 (NC group) patients that underwent health examinations after menopause were selected as controls. All of the patients recruited had normal blood pressures and electrocardiograms, negative for urine proteins, free of symptoms such as fever, chest distress, labored breathing, dizziness etc. Any patients with HbA1c lower than 9\%, underweight, or had normal body weights, were excluded during the selection process. Menopause was defined as follows: natural menopause for more than 12 months; estradiol $<30 \mathrm{pg} / \mathrm{mL}$; FSH $>40 \mathrm{IU} / \mathrm{L}$. The diagnostic criteria for diabetes were obtained from the 1999 diagnostic criteria of WHO for diabetes, i.e., fasting blood-glucose $\geq 7.0 \mathrm{mM}$ and (or) blood glucose $\geq 11.0 \mathrm{mM}$ at $2 \mathrm{~h}$ after glucose load (Wendland et al., 2012). Breast cancer was pathologically confirmed after operation. 


\section{Determination of experimental indexes and methods}

Collection and determination of specimens: A fasting venous blood draw of 10 $\mathrm{mL}$ was performed on all research subjects in the morning (fasting for $8 \mathrm{~h}$ ). Part of the collected blood $(5 \mathrm{~mL})$ was used for determining HbA1c (glycosylated hemoglobin), TG (triglyceride), E2 (estradiol), and Ins (fasting insulin) within $2 \mathrm{~h}$. The remaining $5 \mathrm{~mL}$ was centrifuged and stored at $-70^{\circ} \mathrm{C}$. The above index determination method was in accordance with the instructions provided by the kits (Sigma, CA, and USA). BMI (body mass index) evaluation was as follows: body height and weight was measured, BMI = body weight/ height ${ }^{2}\left(\mathrm{~kg} / \mathrm{m}^{2}\right)$. The steady-state model evaluation method was used to evaluate the insulin resistance index: $($ HOMA-IR $)=($ fasting blood glucose $\mathrm{x}$ fasting insulin $) / 22.5$ (Shaban et al., 2014).

\section{Real-time quantitative PCR detection of miR-221/222 expression in patients' serum}

The reaction mixture for inverse transcription of miRNAs was initially assembled by combining diluted RNA with RT primer working fluid and DEPC water. RNA was denatured for $5 \mathrm{~min}$ at $72^{\circ} \mathrm{C}$, and placed on ice for rapid cooling. The following reverse transcription components were then added (the above mixture; $5 \mathrm{X}$ buffer; $10 \mathrm{mM}$ dNTP; RNase inhibitor; ReverTra Ace). Cycling parameter was as follows: $42^{\circ} \mathrm{C}$ for $1 \mathrm{~h}$ and $95^{\circ} \mathrm{C}$ for $5 \mathrm{~min}$. Amplified cDNAs were cooled on ice and stored.

The expression of miR-221/222 in the serum of patients from all three groups was determined by real time PCR with SYBR ExTaq Mix, and U6 was used as internal reference. The reaction mixture was as follows: SYBR ExTaq Mix II $(12.5 \mu \mathrm{L})$; upstream primer $(1 \mu \mathrm{L})$; downstream primer $(1 \mu \mathrm{L})$; cDNA template $(2 \mu \mathrm{L})$; $\mathrm{dH}_{2} 0(8.5 \mu \mathrm{L})$. The cycling parameters were as follows: $95^{\circ} \mathrm{C}$ for $10 \mathrm{~s} ; 95^{\circ} \mathrm{C}$ for $5 \mathrm{~s}$; at $58^{\circ} \mathrm{C}$ for $30 \mathrm{~s}$; reading the plate; $72^{\circ} \mathrm{C}$ for 30 $\mathrm{s}$; reading the plate; 30 cycles; $2^{\circ} \mathrm{C}$ for $10 \mathrm{~min} ; 55^{\circ} \mathrm{C}$ for $5 \mathrm{~min}$; dissolution curve $55-95^{\circ} \mathrm{C}$, $0.3^{\circ} \mathrm{C} / \mathrm{s}$. The $2^{-\Delta \Delta \mathrm{Ct}}$ method was used to calculate relative expression of the target gene. Primers were designed as shown previously (Gan et al., 2014). All qPCR experimental materials were purchased from the Fermentas Company (Shanghai, China).

\section{Plasmid transfection and luciferase reporter gene assay}

The empty vector pcDNA3.1, the luciferase reporter vector pGL3M, and the recombinant reporter vector pcDNA 3.1-CAV1-WT (or MUT/NC), were transfected with the competent host bacterium DH5a. Plasmid extraction was performed in accordance with product instructions. The final plasmid DNA was identified by double enzyme digestion of $X b a \mathrm{I}$ and $E c o$ RI. The recombinant DNA was submitted to the Hohhot Moore reagent company for sequencing. The vectors and enzymes used in the experiment were purchased from the NEB Company (USA).

pGL3M-CAV1-3'UTR (50 ng) and control plasmid pcDNA3.1 (10 ng) were cotransfected into $293 \mathrm{~T}$ cells using the transfection method mediated by Lipofectamine 2000 (Invitrogen, USA) lipidosome. They were named as follows: experimental group pGL3MWT-CAV1-3'UTR, positive control group pGL3M-MUT-CAV1-3'UTR, and negative control group pGL3M. Enzyme activity was assessed by dual-luciferase reporter assay, and was expressed as the ratio of firefly luciferase activity to the activity of ranilla luciferase. 


\section{Statistical analysis}

SPSS17.0 was used for statistical analysis. All data are reported as means \pm standard deviation. The expression of miR-221/222 in serum was compared between all groups using one-way analysis of variance. Simple correlations were performed using the Pearson correlation analysis. Multiple-factor analyses were conducted with the multiple stepwise regression method. Comparisons between groups were subjected to the Student $t$-tests and chi-square tests. $\mathrm{P}<0.05$ was considered statistically significant.

\section{RESULTS}

\section{General clinical data}

There was no statistical difference in age amongst the patients in the T2DM group, the breast cancer group, the T2DM + breast cancer group, and the normal control group $(\mathrm{P}>$ $0.05)$. BMI, insulin resistance index, $\mathrm{HbAlc}$, and TG were higher in all the diseased groups as compared with those of the healthy people $(\mathrm{P}<0.05)$. HbA1c in the breast cancer group was still within the normal range but was higher than that in the normal control group $(\mathrm{P}<0.05)$; $\mathrm{BMI}$ in the group of T2DM + breast cancer group was lower than that in the T2DM group and the breast cancer group $(\mathrm{P}<0.05)$; the insulin resistance index in the T2DM + breast cancer group was higher as compared with that of the T2DM group and the breast cancer group ( $\mathrm{P}$ $<0.05$ ); the levels of estradiol and fasting insulin in healthy people with a normal body mass index were lower than those in the diabetes and breast cancer patients with a high body mass index $(\mathrm{P}<0.05)$; the levels of estradiol and fasting insulin in the breast cancer group were higher than those in the diabetes group and the diabetes + breast cancer group $(\mathrm{P}<0.05)$, as shown in Table 1.

Table 1. General clinical data of recruited patients.

\begin{tabular}{l|l|l|l|l|l|c|c}
\hline Group & Age (years) & BMI $\left(\mathrm{kg} / \mathrm{m}^{2}\right)$ & HOMA-IR & HbA1c $(\%)$ & TG $(\mathrm{mM})$ & $\mathrm{E} 2(\mathrm{pg} / \mathrm{mL})$ & $\mathrm{Ins}(\mathrm{IU} / \mathrm{mL})$ \\
\hline NC & $59.78 \pm 11.23$ & $20.12 \pm 1.69$ & $1.59 \pm 0.33$ & $4.56 \pm 0.45$ & $0.80 \pm 0.28$ & $14.48 \pm 3.33$ & $7.58 \pm 2.28$ \\
\hline DM & $60.79 \pm 11.11$ & $28.88 \pm 1.18^{*}$ & $7.38 \pm 0.39^{*}$ & $7.60 \pm 0.33^{*}$ & $2.70 \pm 0.40^{*}$ & $22.58 \pm 3.22^{*}$ & $12.57 \pm 3.08^{*}$ \\
\hline BC & $61.89 \pm 9.33$ & $29.24 \pm 1.32^{*}$ & $7.50 \pm 0.44^{*}$ & $5.30 \pm 0.33^{* \#}$ & $2.45 \pm 0.35^{*}$ & $25.50 \pm 3.38^{*}$ & $16.62 \pm 2.88^{* *}$ \\
\hline DM complicated by BC & $61.13 \pm 10.58$ & $27.24 \pm 1.37^{*}$ & $8.20 \pm 0.58^{*}$ & $7.57 \pm 0.36^{* a}$ & $2.45 \pm 0.38^{* \mathrm{~b}}$ & $20.66 \pm 3.47^{* a}$ & $13.38 \pm 2.98^{* a}$ \\
\hline
\end{tabular}

$* \mathrm{P}<0.05$ between the patient group and normal group; ${ }^{*} \mathrm{P}<0.05$ between the $\mathrm{BC}$ group and the DM group; ${ }^{a} \mathrm{P}<$ 0.05 between the $\mathrm{DB}$ group and the $\mathrm{BC}$ group; ${ }^{\mathrm{b}} \mathrm{P}<0.05$ between the $\mathrm{DB}$ group and the $\mathrm{DM}$ group. $\mathrm{NC}=$ normal control group; $\mathrm{DM}=$ diabetes group; $\mathrm{BC}=$ breast cancer group; $\mathrm{BMI}=$ body mass index; HOMA-IR = insulin resistance index; HbA1c = glycosylated hemoglobin; TG = triglyceride; E2 = estradiol; Ins = fasting insulin.

\section{Expression of miR-221/222 in the serum of diabetes patients, breast cancer patients, and normal subjects}

There was a significant difference in the expression of miR-221/222 amongst the four groups $(\mathrm{F}=432.55,344.09 ; \mathrm{P}<0.001)$; there were statistical differences amongst all groups based on further pairwise comparisons $(\mathrm{P}<0.05)$; the expression level of miR-221/222 in the patients with post-menopausal breast cancer was higher as compared with T2DM patients ( $\mathrm{P}$ $<0.05)$ and lower as compared with patients with T2DM + post-menopausal breast cancer $(\mathrm{P}<0.05)$; the expression level of miR-221 (Figure 1A) and miR-222 (Figure 1B) in T2DM patients was higher than that in the normal subjects $(\mathrm{P}<0.05)$. 
A

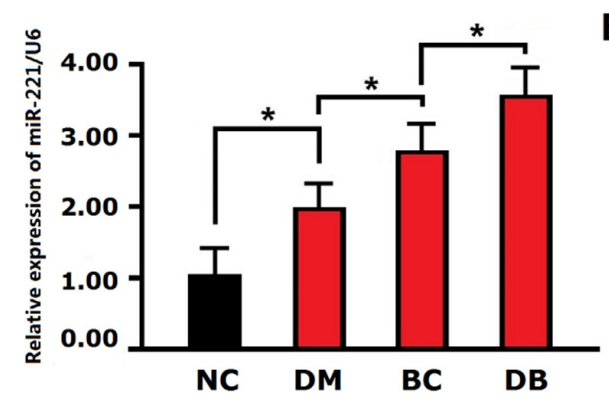

B

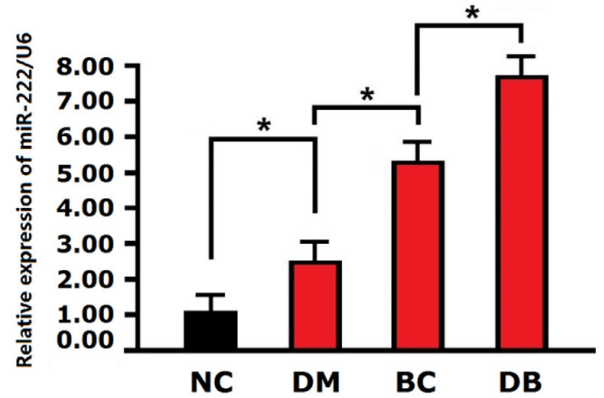

Figure 1. Relative expression of miR-221/222 in each group. A. Expression of miR-221 relative to U6; B. expression of miR-222 relative to U6; *P $<0.05$; NC: normal group; DM: diabetes group: BC: breast cancer group; DB: diabetes with breast cancer.

\section{Relationship between miR-221/222, BMI, TG, HbA1c, and HOMA-IR}

Based on the Pearson correlation analysis, BMI, TG, HbAlc, HOMA-IR, and relative expression of miR-221/222 exhibited positive correlation $(\mathrm{r}$ miR-221 $=0.457,0.678,0.480$, $0.628 ; \mathrm{r}$ miR-222 $=0.645,0.819,0.567,0.739 ; \mathrm{P}<0.01)$. Multiple linear stepwise regression analysis was used with miR-221 and miR-222 serving as dependent variables. The regression analysis with correlated variables MI, TG, HbA1c, and HOMA-IR as the independent variables indicated that BMI and HOMA-IR were independent influencing factors of miR-221 and miR222. TG was the independent influencing factor of miR-222 and miR-221 (Tables 2 and 3).

Table 2. Multivariate linear regression analysis of miR-221.

\begin{tabular}{l|c|c|c|c|c}
\hline Independent variable & Regression coefficient & Standard error & Standard regression & T value & P value \\
\hline BMI & -0.445 & 0.094 & -0.628 & -4.905 & 0.000 \\
\hline TG & 1.234 & 0.458 & 0.375 & 2.695 & 0.063 \\
\hline HbA1C & -0.210 & 0.159 & -0.115 & -1.311 & 0.190 \\
\hline HOMA-IR & 1.055 & 0.169 & 0.986 & 4.158 & 0.000 \\
\hline
\end{tabular}

BMI: body mass index; TG: triglyceride: HbA1c: glycosylated hemoglobin; HOMA-IR: insulin resistance index.

Table 3. Multivariate linear regression analysis of miR-222.

\begin{tabular}{l|c|c|c|c|c}
\hline Independent variable & Regression coefficient & Standard error & Standard regression & T value & P value \\
\hline BMI & -2.059 & 0.549 & -0.370 & -3.600 & 0.000 \\
\hline TG & 8.745 & 2.858 & 0.329 & 3.059 & 0.004 \\
\hline HbA1C & -1.210 & 1.040 & -0.079 & -1.210 & 0.310 \\
\hline HOMA-IR & 7.532 & 1.020 & 0.911 & 7.328 & 0.000 \\
\hline
\end{tabular}

BMI: body mass index; TG: triglyceride: HbA1c: glycosylated hemoglobin; HOMA-IR: insulin resistance index.

\section{Verifying the potential target gene CAV1 by the luciferase reporter assay}

MiR-221 and 222 had 18 and 16 sequences respectively, which is consistent with WT-CAV1-3'UTR (Figure 2A). In the mutant MUT-CAV1, where base sequences were disrupted, the luciferase reporter assay of the transfected 293T cells indicated that there were no significant changes in pGL3M-MUT-CAV1-3'UTR and pGL3M-WT-CAV1-3'UTR in the negative control group compared with pGL3M in the vacant plasmid group, as shown in Figure 
2B. There were no significant changes in viability of the MUT group while the fluorescence intensity in the WT group decreased significantly following addition of miR-221 (miR-222) mimics (Figure 2B). Taken together, these results indicated that miR-221 (miR-222) was able to bind with specific sequences in the promoter region of WT-CAV1-3'UTR. MiR-221 (miR222) did not function after these specific sequences on the promoter were altered.

A

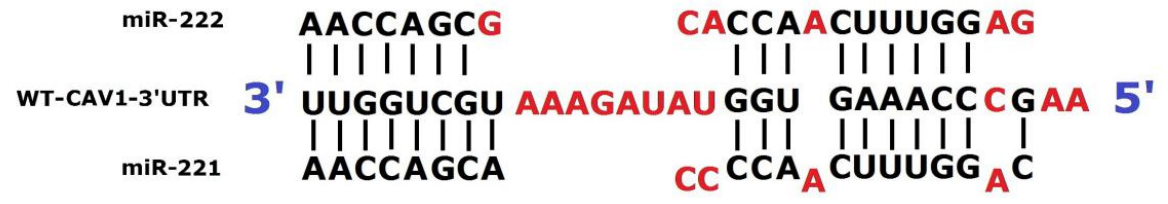

B
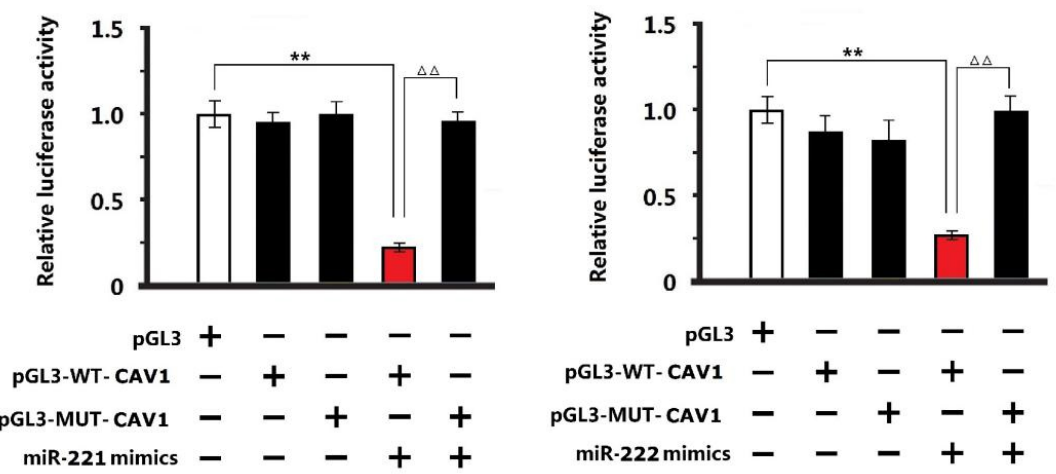

Figure 2. Verification of the miR-221 (miR-222) target gene, CAV1, with luciferase reporter assay. A. miR-221 and 222 sequences, as compared with WT-CAV1-3'UTR; B. relative fluorescent intensity of MUT and WT groups following addition of miR-221 (miR-222) mimics, ${ }^{*} \mathrm{P}<0.01$.

\section{DISCUSSION}

Breast cancer is a heterogeneous hormone-dependent malignant tumor. There is a significant rise in the incidence of post-menopausal breast cancer in female T2DM patients. Furthermore, the incidence of T2DM also rises in patients with post-menopausal breast cancer, suggesting that these diseases have common pathogenic factors (Crujeiras et al., 2013). Research both at home and abroad has indicated that miRNAs participate in the pathogenesis of Type-2 diabetes and breast cancer. The expression of MiR-221/222 is up-regulated in rats and humans with fatty liver disease. It is also highly expressed in the breast cancer tissue, and is secreted into blood through exocytosis. In addition, the expression of miR221/222 was detected in patients with T2DM, patients with post-menopausal breast cancer, and the patients with both T2DM and post-menopausal breast cancer. As expected, its expression in the serum of the patients with T2DM alone and those with post-menopausal breast cancer alone is higher in the serum of the normal people. Additionally, its expression level was observed to be the highest when both diseases exist. This further suggests that MiR-221/222 may participate in the incidence of T2DM with post-menopausal breast cancer complications. 
In this study, we tried to determine whether there are correlation between BMI, HOMA-IR, and miR-221/222. Results indicated that BMI, HOMA-IR, and miR221/222 exhibit positive correlations. BMI and HOMA-IR are the independent influencing factors of miR221/222. The expression of miR-221/222 is present in the serum of the patients with T2DM and the patients with post-menopausal breast cancer. The expression of miR-221/222 is higher when both diseases are present (Xuan et al., 2015). The above research results indicate that miR-221/222 not only stems from tissues such as liver fat, but is also present in breast cancer tissues. Therefore, factors such as diabetes should be considered when miR-221/222 is found serum during molecular diagnosis of breast cancer.

Previous research indicates that down-regulation of CAV1 may cause insulin resistance; blood glucose is still within the normal range but higher than that of normal people due to the fact that the pancreatic $\beta$ cells have an excellent compensatory functions. Finally, abnormality in glucose tolerance and even diabetes may occur as compensatory functions of pancreatic $\beta$ cells decreases with age (Bitar et al., 2013). This experiment has demonstrated that CAV1 is a target gene of miR-221/222. Thus, the regulation of CAV1 by miR-221/222 may contribute to insulin resistance in post-menopausal breast cancer. We also found that TG and HOMA-IR in the patients with post-menopausal breast cancer were higher than those in normal people; there was no significant difference in abnormal lipid metabolism and HOMA-IR between patients with post-menopausal breast cancer and the patients with T2DM. TG was found to be an independent influencing factor of miR222. Our results suggest that insulin resistance in post-menopausal breast cancer can lead to elevated blood glucose level and susceptibility to T2DM. Hence, post-menopausal breast cancer is also one of the risk factors of T2DM. Conversely, obesity has also been shown to be a risk factor for T2DM and post-menopausal breast cancer, and can lead to a lipid metabolism disorders (Jedrzejuk and Milewicz, 2005).

High estrogen levels were observed in overweight or obese female T2DM patients in this study. Binding of estrogen can stimulate proliferation of mammary glandular cells and gene expression; it can also cause DNA damage by formation of oxidative products, and participate in the initiation and progression of breast cancer (Yubero-Serrano et al., 2015). High expression of miR-221/222 in breast cancer tissues causes insulin resistance and increases the metabolic load on pancreatic $\beta$ cells. This leads to elevated blood glucose levels, and T2DM finally occurs when compensation becomes insufficient (Díaz-Villaseñor et al., 2013). Therefore, miR-221/222 and estrogen may jointly participate in pathogenesis of T2DM with post-menopausal breast cancer in overweight or obese females.

In conclusion, the relative expression of serum miR-221/222 and HOMA-IR exhibited a positive correlation in the T2DM group, indicating that miR-221/222 may be a contributing factor to insulin resistance. Highly-expressed miR-221/222 activates estrogen receptors by down-regulation of CAV1, leading to insulin resistance-induced hyperinsulinemia, and increased expression of estrogens and their receptors in the serum (Rippe et al., 2012). Therefore, miR$221 / 222$ and estrogens may jointly contribute to T2DM with post-menopausal breast cancer in overweight or obese females. Down-regulation of CAV1 in the liver and fat tissues mainly causes hyperinsulinemia. Down-regulation of CAV1 in the skeletal muscles mainly causes reduced glucose uptake and increased blood glucose. Abnormal expression of CAV1 can not only cause insulin resistance and hyperglycemia, but also participates in formation of breast cancer and abnormal expression of metastasized CAV1. It is closely associated with the occurrence, progression, and prognosis of breast cancer (Tan et al., 2012; Ma et al., 2013). The mutation rate of the pre-menopausal CAV1 is higher than that of the post-menopausal CAV1, but the morbidity 
of post-menopausal breast cancer is higher as compared with that of pre-menopausal breast cancer. This suggests that the occurrence of breast cancer arises from multiple factors, and that miR-221/222 is one of such factors (Banin Hirata et al., 2014).

Highly expressed miR-221/222 inhibits the expression of CAV1 and causes insulin resistance. Thus, miR-221/222 may be a contributing factor of T2MD with post-menopausal breast cancer. It may also serve as a target for therapeutic treatments as well as be used as molecular markers for diagnosis and prognosis of the disease.

\section{Conflicts of interest}

The authors report no conflicts of interest.

\section{ACKNOWLEDGMENTS}

Research supported by grants from the government or any other business organizations.

\section{REFERENCES}

Banin Hirata BK, Oda JM, Losi Guembarovski R, Ariza CB, et al. (2014). Molecular markers for breast cancer: prediction on tumor behavior. Dis. Markers 2014: 513158. http://dx.doi.org/10.1155/2014/513158

Bitar MS, Abdel-Halim SM and Al-Mulla F (2013). Caveolin-1/PTRF upregulation constitutes a mechanism for mediating p53-induced cellular senescence: implications for evidence-based therapy of delayed wound healing in diabetes. Am. J. Physiol. Endocrinol. Metab. 305: E951-E963. http://dx.doi.org/10.1152/ajpendo.00189.2013

Chen W, Chen Y, Qin L, Li A, et al. (2011). Transcription factor Sp1 is essential for the regulation of the porcine caveolin-1 gene. DNA Cell Biol. 30: 491-497. http://dx.doi.org/10.1089/dna.2010.1202

Crujeiras AB, Díaz-Lagares A, Carreira MC, Amil M, et al. (2013). Oxidative stress associated to dysfunctional adipose tissue: a potential link between obesity, type 2 diabetes mellitus and breast cancer. Free Radic. Res. 47: 243-256. http://dx.doi.org/10.3109/10715762.2013.772604

Dentelli P, Traversa M, Rosso A, Togliatto G, et al. (2014). miR-221/222 control luminal breast cancer tumor progression by regulating different targets. Cell Cycle 13: 1811-1826. http://dx.doi.org/10.4161/cc.28758

Díaz-Villaseñor A, Cruz L, Cebrián A, Hernández-Ramírez RU, et al. (2013). Arsenic exposure and calpain-10 polymorphisms impair the function of pancreatic beta-cells in humans: a pilot study of risk factors for T2DM. PLoS One 8: e51642. http://dx.doi.org/10.1371/journal.pone.0051642

Du C, Li Z, Wang S, Zhou Z, et al. (2014). Tongshu capsule down-regulates the expression of estrogen receptor $\alpha$ and suppresses human breast cancer cell proliferation. PLoS One 9: e104261-e104261. http://dx.doi.org/10.1371/journal. pone. 0104261

Gallagher EJ, Fierz Y, Vijayakumar A, Haddad N, et al. (2012). Inhibiting PI3K reduces mammary tumor growth and induces hyperglycemia in a mouse model of insulin resistance and hyperinsulinemia. Oncogene 31: 3213-3222. http://dx.doi.org/10.1038/onc.2011.495

Gan R, Yang Y, Yang X, Zhao L, et al. (2014). Downregulation of miR-221/222 enhances sensitivity of breast cancer cells to tamoxifen through upregulation of TIMP3. Cancer Gene Ther. 21: 290-296. http://dx.doi.org/10.1038/cgt.2014.29

García MA, Peña D, Alvarez L, Cocca C, et al. (2010). Hexachlorobenzene induces cell proliferation and IGF-I signaling pathway in an estrogen receptor $\alpha$-dependent manner in MCF-7 breast cancer cell line. Toxicol. Lett. 192: 195-205. http://dx.doi.org/10.1016/j.toxlet.2009.10.026

Hwang MS, Yu N, Stinson SY, Yue P, et al. (2013). miR-221/222 targets adiponectin receptor 1 to promote the epithelialto-mesenchymal transition in breast cancer. PLoS One 8: e66502-e66502. http://dx.doi.org/10.1371/journal. pone. 0066502

Jedrzejuk D and Milewicz A (2005). Consequences of menopause in women with diabetes mellitus - a clinical problem. Gynecol. Endocrinol. 21: 280-286. http://dx.doi.org/10.1080/09513590500378964

Joglekar M, Elbazanti WO, Weitzman MD, Lehman HL, et al. (2015). Caveolin-1 mediates inflammatory breast cancer cell invasion via the Akt1 pathway and RhoC GTPase. J. Cell. Biochem. 116: 923-933. http://dx.doi.org/10.1002/jcb.25025 
Ma X, Liu L, Nie W, Li Y, et al. (2013). Prognostic role of caveolin in breast cancer: a meta-analysis. Breast 22: 462-469. http://dx.doi.org/10.1016/j.breast.2013.03.005

Reinbothe S, Larsson AM, Vaapil M, Wigerup C, et al. (2014). EPO-independent functional EPO receptor in breast cancer enhances estrogen receptor activity and promotes cell proliferation. Biochem. Biophys. Res. Commun. 445: 163-169. http://dx.doi.org/10.1016/j.bbrc.2014.01.165

Rippe C, Blimline M, Magerko KA, Lawson BR, et al. (2012). MicroRNA changes in human arterial endothelial cells with senescence: relation to apoptosis, eNOS and inflammation. Exp. Gerontol. 47: 45-51. http://dx.doi.org/10.1016/j. exger.2011.10.004

Roh E, Kwak SH, Jung HS, Cho YM, et al. (2015). Serum aryl hydrocarbon receptor ligand activity is associated with insulin resistance and resulting type 2 diabetes. Acta Diabetol. 52: 489-495. http://dx.doi.org/10.1007/s00592-014$\underline{0674-z}$

Shaban N, Kenno KA and Milne KJ (2014). The effects of a 2 week modified high intensity interval training program on the homeostatic model of insulin resistance (HOMA-IR) in adults with type 2 diabetes. J. Sports Med. Phys. Fitness 54: 203-209.

Tan Z, Zhou LJ, Mu PW, Liu SP, et al. (2012). Caveolin-3 is involved in the protection of resveratrol against high-fatdiet-induced insulin resistance by promoting GLUT4 translocation to the plasma membrane in skeletal muscle of ovariectomized rats. J. Nutr. Biochem. 23: 1716-1724. http://dx.doi.org/10.1016/j.jnutbio.2011.12.003

Tawfik SH, Mahmoud BF, Saad MI, Shehata M, et al. (2015). Similar and additive effects of ovariectomy and diabetes on insulin resistance and lipid metabolism. Biochem. Res. Int. 2015: 567945. http://dx.doi.org/10.1155/2015/567945

Wen F, Yang Y, Jin D, Sun J, et al. (2014). MiRNA-145 is involved in the development of resistin-induced insulin resistance in HepG2 cells. Biochem. Biophys. Res. Commun. 445: 517-523. http://dx.doi.org/10.1016/j.bbrc.2014.02.034

Wendland EM, Torloni MR, Falavigna M, Trujillo J, et al. (2012). Gestational diabetes and pregnancy outcomes-a systematic review of the World Health Organization (WHO) and the International Association of Diabetes in Pregnancy Study Groups (IADPSG) diagnostic criteria. BMC Pregnancy Childbirth 12: 23. http://dx.doi. org/10.1186/1471-2393-12-23

Xuan H, Xue W, Pan J, Sha J, et al. (2015). Downregulation of miR-221, -30d, and -15a contributes to pathogenesis of prostate cancer by targeting Bmi-1. Biochemistry 80: 276-283. http://dx.doi.org/10.1134/S0006297915030037

Yubero-Serrano EM, Woodward M, Poretsky L, Vlassara H, et al.; AGE-less Study Group (2015). Effects of sevelamer carbonate on advanced glycation end products and antioxidant/pro-oxidant status in patients with diabetic kidney disease. Clin. J. Am. Soc. Nephrol. 10: 759-766. http://dx.doi.org/10.2215/CJN.07750814

Zhang Y, Cheng JC, Huang HF and Leung PC (2013). Homeobox A7 stimulates breast cancer cell proliferation by upregulating estrogen receptor-alpha. Biochem. Biophys. Res. Commun. 440: 652-657. http://dx.doi.org/10.1016/j. bbrc.2013.09.121

Zhu J, Zhao C, Kharman-Biz A, Zhuang T, et al. (2014). The atypical ubiquitin ligase RNF31 stabilizes estrogen receptor $\alpha$ and modulates estrogen-stimulated breast cancer cell proliferation. Oncogene 33: 4340-4351. http://dx.doi. org/10.1038/onc. 2013.573 